Progress in Aerospace and Aviation Technology

\title{
Issues of Designing a Model Adaptive Controller Without a State Observer
}

\author{
Najmaddin Abo Mosali ${ }^{*}$, Syariful Syafiq Shamsudin ${ }^{1}$ \\ ${ }^{1}$ Universiti Tun Hussein Onn Malaysia UTHM, 86400 Parit Raja, Johor, MALAYSIA \\ *Corresponding Author
}

DOI: https://doi.org/10.30880/paat.2021.01.01.001

Received 24 September 2021; Accepted 11 November 2021; Available online 21 December 2021

\begin{abstract}
It can be challenging to develop a controller using conventional techniques for a plant with a linear or nonlinear dynamical system or model uncertainty. Model adaptive control is a new alternative to classical control techniques and a simple way to update controller parameters. Because model reference adaptive control is unable to anticipate the state in real time if the state observer is not designed with, we will review some of the most major disadvantages of the most commonly used design techniques without state observer in this work.
\end{abstract}

Keywords: Controller, model adaptive controller, state observer, plant

\section{Introduction}

Since the early 1950s, adaptive control systems have been used and its development period was accelerated during the 1960s in order to demonstrate its stability and use in control system operation. An adaptive controller is necessary in process control because of its benefits, which include: a) the presence of variation in process dynamics where system parameters may change throughout operation, b) the variation of disturbances, and c) the resulting process efficiencies[8]. Gain Scheduling, Self-tuning Regulator, Dual Control, and Model Reference Adaptive Control are examples of adaptive controllers (MRAC). One of the most extensively utilized control strategies for designing sophisticated control systems for higher performance and accuracy is adaptive control[4]. It is a series of approaches that give a systematic approach for automatic controller adjustment in real time in order to maintain a desired level of control system performance when the plant dynamic model parameters change over time or are unknown[2]. Adjusting the controller's control law to deal with the reality that the variables of the system being managed change dramatically owing to changes in environmental conditions or changes in the system itself is what adaptive control is all about. This strategy is based on an existing organism's core trait of adaptation[2]. Adaptive controllers are particularly useful in situations where parameter changes and environmental changes occur frequently. Figure 1 depicts the principle design of a control system as well as an adaptive control system. 


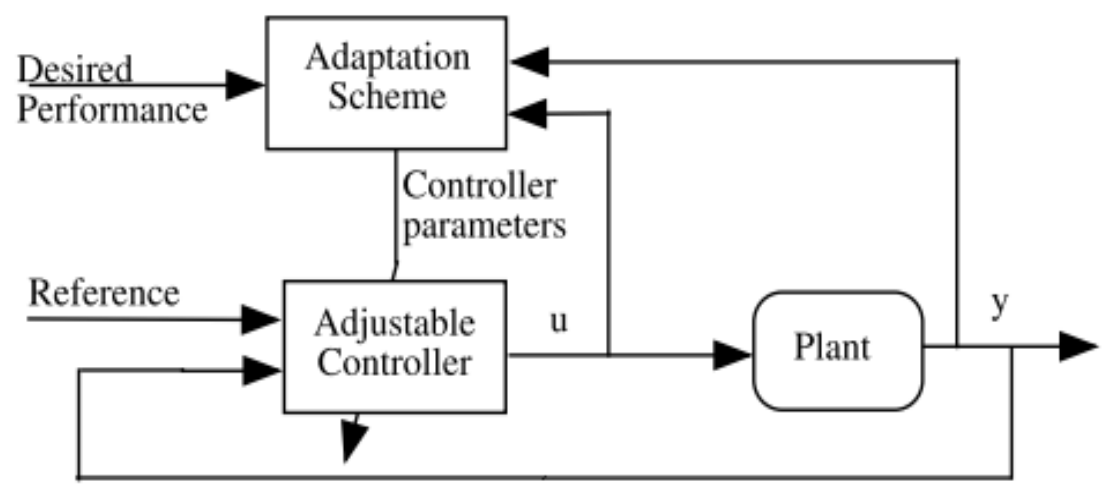

Fig. 1 - a principle and adaptive control system[1]

\section{Adaptive Control}

Early on in the development of modern control theory, it was shown that a fixed controller could not provide proper system behavior in all scenarios. It's challenging to build a fixed controller that always meets the required requirements, especially when the process to be governed comprises unknown or time-varying variables. The fact that adaptive algorithms' evolution is known to be reliant on some external, quantifiable condition spurred a great interest in adaptive algorithms in the late 1950s. For example, an aircraft's reaction to the pilot's directions is proportionate to its height. Because the craft's behavior changes with altitude, a controller can be constructed for any altitude and the closed loop will always match the criteria. The controller may be modified in real time by sensing altitude[2]. Adaptive Control approaches offer a method for automatically tuning controller parameters on-the-fly. Approximations of several nonlinear stochastic control issues can also be shown (not solvable in practice). When plant (disturbance) model parameters are unknown or vary, the goal of adaptive control is to achieve and maintain an acceptable level of performance. Plant (disturbance) model parameters may be unknown or time-varying, therefore high-performance control systems may necessitate accurate controller tuning.

\subsection{Model Reference Adaptive Control Systems}

One adaptive control approach with a lot of promise and capacity to cope with complex systems in the face of significant both uncertainties and changes in relation to system parameters is model reference adaptive controller (MRAC) [10]. MRAC is a direct adaptive approach with a mechanism for modifying controller settings [4]. In 1958, Whitacker was the first to propose it [1]. To optimize system efficiency, plant flexibility, and track anticipated outcomes, post a temperature profile that matches the raw material to be extracted. A model reference's most important role is to signal the controller's intended output, and the plant's unknown parameters are updated using the adaptation law whereby the closed loop system's output should reflect the reference model's output. In other words, tracking mistake should be reduced to a minimum. The results demonstrated that model reference control was successfully implemented in the appropriate processes [10], such as temperature control in a variety of industrial applications. In the steam distillation process, the performance of the MRAC was demonstrated in real-time and simulated conditions using Lyapunov and MIT approaches [4]. Minimal SSE and RMSE were obtained in real-time control whenever the integral limitation was eliminated from the MRAC Lyapunov and MRAC MIT control structures [4]. In relationto to MRAC literature, a study carried out a simulation of the MRAC in terms of using it to manage steam temperature in the process of distillation for the extraction systems of oil essential, and it was found that steam temperature is considered to be among the most critical factors, impacting the composition of essential oil production in terms of quantity and quality [11]. The approach of MRAC utlises estimation through online parameter using recursive least squares. The experimental result supported the validation of MRAC's efficacy in the process of distillation, as well as the stability of this system [11]. The model is mentioned. Adaptive control's inner and outer loops are the inner and outer loops, respectively. The inner loop is a general feedback signal from the process to the controller, whilst the outer loop is an adjustment mechanism which regulates and sends back to the controller the error signal (the difference between the process output and the model reference). MRAC's key concept is that the process output responds in just such a way that the model reference is followed. This is performed by modifying the controller settings with an adjustment mechanism, leading the error to converge to zero. The Lyapunov function is utilized in this work to fine-tune in terms of the zero error and, more importantly, to ensure system stability [9].

The bleaching temperature control approach is MRAC, with the required transient specification supplied in terms of reference model [1]. As a result, the controller is changed continually; hence, the output of the system approaches asymptotically the reference model output and provides the required response to the command signal. It can, for example, operate a system that is subject to parameter and/or environmental changes[12]. 


\section{Components of Model Reference Adaptive Control}

The three components of model reference adaptive control are:

3.1 Model of Reference: It is used to indicate the adaptive control system's optimal reaction to an external command. It should correspond to the performance requirements for control jobs. The adaptive control system should be able to achieve the ideal behavior indicated by the reference model, and this paper used the critically damped second order system as the reference model [13].

3.2 Controller: A set of variables that can be altered is typically used to parameterize it. In this work, the controller law is defined utilizing two parameters: 1 and 2. The control law is considered to be a linear regarding the configurable parameters (linear parameterization). Adaptive controller design is frequently necessitating linear parameterization which aims to construct an adaptation mechanism, and this ensures stability as well as tracking convergence. In terms of the values of these control parameters, they are mostly controlled by the process of adaptation gain, which also works to change the control algorithm related to the adaptation mechanism [13].

3.3 Adaptation Mechanism: This mechanism is utilized to alter the control law's parameters. The adaptation law looks for factors that will give the plant the same response as the reference model. It is intended to ensure the control system's stability as well as the tracking error's reduction to zero. The development of the adaptation mechanism can be achieved through the use of mathematical techniques, including the MIT rule, and augmented error theory, Lyapunov theory. The MIT rule is employed in this study for this purpose[13].

\section{Methods of Designing MRAC}

The gradient approach and the Lyapunov method are two most used of the various methods for designing MRAC. In the following part, we will go over these two techniques.

\subsection{Gradient Method}

It's also named as MIT rule due to fact that was developed in Massachusetts Institute of technology. The main objective of this technique is to make the output's plant track the reference model. So, Design controller drives the plant response to mimic ideal response. In MIT rule, the cost function $J(\theta)=\frac{1}{2} e^{2}$ is minimized.

The associated equation to MIT rule is

$$
\frac{d \theta}{d t}=-\gamma \frac{\partial J}{\partial \theta}=-\gamma \mathrm{e} \frac{\partial \mathrm{e}}{\partial \theta}
$$

Where $\theta$ is the controller parameter; e is the error between the plant and the reference model; $\gamma$ is the adaption gain; $\frac{\partial \mathrm{e}}{\partial \theta}$ is called the sensitivity derivative, which indicates how the error is influenced by the controller parameter, $\theta$.

Supposing that the cost function $J(\theta)=|e|$ then the adjustment factor depends, among other variables, on sign (e) as shown:

$$
\frac{d \theta}{d t}=-\gamma \frac{\partial J}{\partial \theta}=-\gamma \mathrm{e} \frac{\partial \mathrm{e}}{\partial \theta}=-\gamma \frac{\partial \mathrm{e}}{\partial \theta} \operatorname{sign}(e)
$$

Where sign $(e)=\left\{\begin{array}{lll}+1 & \text { if } & e \geq 0 \\ -1 & \text { if } & e<0\end{array}\right\}$

\subsection{Lyapunov Method}

Because MRAC systems are nonlinear and time-varying, a linear stability analysis technique cannot be used. Lyapunov's direct technique is a well-known method for ensuring the overall stability of the adaptive system. If a Lyapunov function $\mathrm{V}(\mathrm{x})$ exists that satisfies the following conditions, a system has a uniform asymptotically stable equilibrium $\mathrm{x}=0$ [2]:

$$
\begin{gathered}
V(x)>0 \text { for } x \neq 0 \quad \text { (positive definite) } \\
V(x)<0 \text { for } x \neq 0 \text { (negative definite) } \\
V(x) \rightarrow \infty \text { for } ? x \text { ? } \rightarrow \infty \\
V(0)=0
\end{gathered}
$$

As a result, the Lyapunov function $\mathrm{V}$ (which may be thought of as an energy function) must decrease over time. The stability requirements are directly turned into an adaptive rule using Lyapunov's technique in adaptive system design, as 
shown below[2]. The error equation, which describes the differential equation of the error signal e (which could be the output error $y \mathrm{p}-\mathrm{ym}$ or the state error $\mathrm{zp}-\mathrm{xm}$ ), is the first stage. Although there is a lot of freedom in the derivation of the error equation, it should be represented as a known linear transfer function controlled by a nonlinear input component in order to be useful. The reference model transfer function is usually included in the linear component. As a result of this need, the error equation takes on a more or less standard form[2]. Second, as a function of both the signal and parameter errors, a Lyapunov function is chosen. This decision is based on a limited set of system states, which includes the signal error vector e $\mathrm{xp}-\mathrm{xm}$ and the parameter error vector $=-*$. The Lyapunov function $\mathrm{V}$ is chosen in its most basic form as:

$$
V=e^{\wedge} T P e+\phi T \Gamma-1 \phi
$$

\section{Advantage of Model Reference Adaptive Control}

The fundamental benefit of an adaptive controller system is that it can react to any changes in a control system's parameters. As a result, issues in determining acceptable control rules that are satisfactory throughout a wide range of operating situations must be overcome.

\section{Disadvantages of Adaptive Control}

Several disadvantages of the adaptive control are discussed on the following prospective.

1. Computational demand and complexity: It has the property of being able to breakdown the control law's detailed structure into an online parameter estimator and an online control law synthesis. In comparison to fixed gain control techniques, this necessitates additional computational effort. Furthermore, for fast dynamics systems with high sampling rates, highly fast processors will be required to execute all of the necessary calculations for the system's control signals [2].

2. Stability and convergence issues: For adaptive control, the process of proving the system stability and convergence is quit complex. Most Stability analyses were based on the assumption that the system order was equal to the assumed model order [2].

3. Accuracy of the parameter estimator: Because it decides the control gain parameter to be employed, the accuracy of the estimated parameters is crucial for the adaptive controller's successful performance. However, there are a number of concerns that must be addressed in order to produce a precise parameter estimation result. Closed loop parameter estimation is one of them. Due to the link between the disturbances and the control signal, closed-loop parameter estimation and tracking for time-varying plants is a difficult system identification problem [2].

\section{State Estimation}

One of the problems of model reference adaptive control is to estimate the state in real time .some methods of state estimation are discussed in the following subtitles:

\subsection{Observer Design}

Observer design is typically regarded as a "graduate-level topic" and taught in "a graduate-level control" engineering course. However, "full-order" and even "reduced-order" observers are covered in the most current editions of numerous textbooks related to "classic undergraduate control system" [14]. The real system is assumed to be a deterministic, linear time-invariant (LTI), continuous control system that is observable and controllable, but whose internal states cannot be determined directly [6]. The plant input and output signals are used by observers to build a state estimate, which is then used to close the control loop [15]. The observer's job is to generate an approximation of the real system's true state $x(t)$. It is acceptable to suppose that there will be some mistake in the estimate at first, but the error should diminish over time [6].

\subsection{System Controllability and Observability}

If a control input exists that changes any state of the system to zero in limited time, the system is said to be controllable. A LTI system can be proved to be controllable if and only if its controllability matrix (2.4) has complete rank [6]. Its rank is equal to the number of states [16]. Note that using the commands $\operatorname{rank}(\operatorname{ctrb}(\mathrm{A}, \mathrm{B}))$ or $\operatorname{rank}(\operatorname{ctrb}(\operatorname{sys}))$ in MATLAB, you may quickly compute the rank of the controllability matrix of an LTI system [6]. 
A system is said to be observable if the initial state $\mathrm{x}\left(\mathrm{t}_{0}\right)$ might be obtained by utilizing the system output, (y) $t$, over some finite time $t_{0} \leq t \geq t_{f}$. Mathematically, a LTI system is observable only when the observability matrix, has a full rank. Its rank is equal to the number of states[16]. Note here also that in MATLAB this can easily be checked by the command $\operatorname{rank}(\operatorname{obsv}(\mathrm{A}, \mathrm{C}))$ or $\operatorname{rank}(\mathrm{obsv}(\mathrm{sys}))[6]$. The block diagram of system and observer models.is shown in Figure 2.2 below.

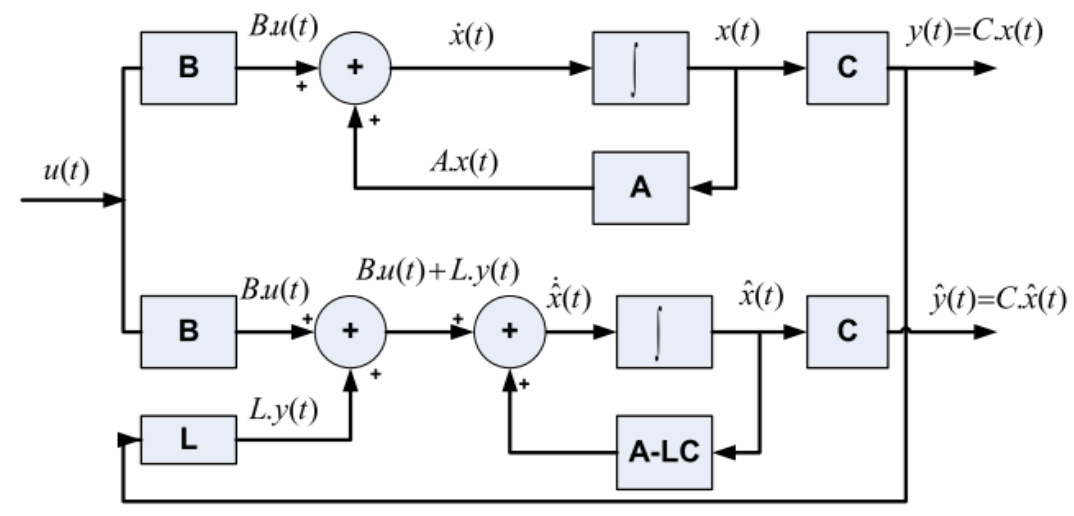

Fig. 2 - Block diagram of system and observer models[6]

\section{Performance Evaluation}

Step response measurements are taken in order to evaluate the controller. The rising time, percentage overshoot, and settling time are the most common metrics used to assess performance [10]. The controller will respond slowly if the rising time is longer. The quality of the controller can be affected by percentage overshoot, whereas settling time is the time it takes for transients to settle. Another measurement technique used by the controller is error performance evaluation. Mean square error (MSE) and root mean square error (RMSE) are two different forms of error analysis[17]. The MSE and RMSE are used to analyze controller performance in steady state.

\section{Conclusion}

Finally, there are a variety of methods for designing model reference adaptive control, such as MIT and others. However, because model reference adaptive control is unable to predict the state in real time without state observer which will be capable of reproduce the state. These are the strategies that are capable of improving MRAC's ability to solve any problem in real time.

\section{References}

[1] S. A. Billings, H. B. Jamaluddin, and S. Chen, "A comparison of the backpropagation and recursive prediction error algorithms for training neural networks," Mech. Syst. Signal Process., vol. 5, no. 3, pp. 233-255, 1991.

[2] S. I. D. Landau and A. Karimi, “Adaptive Control,” pp. 1-81, 2009

[3] "Butler, H. (1990). Model reference adaptive control from theory to practice. Prentice Hall New York." .

[4] N. Kasuan, M. H. A. Jalil, M. Hezri, F. Rahiman, and M. N. Taib, "A Discrete Model Reference Adaptive Control for Temperature Tracking in Steam Distillation Process,” pp. 6-8, 2015

[5] P. Jain and N. M.J, "Design of a Model Reference Adaptive Controller Using Modified MIT Rule for a Second Order System,” Adv. Electron. Electr. Eng., vol. 3, no. 4, pp. 477-484, 2013

[6] T. Ionescu and R. Monopoli, "Discrete model reference adaptive control with an augmented error signal," Automatica, vol. 13, no. 5, pp. 507-517, 1977

[7] L. Sellami, "Simulink Model of a Full State Observer for a DC Motor Position, Speed, and Current," no. 3

[8] M. H. A. Jalil, M. N. Taib, M. H. F. Rahiman, N. N. Nordin, and N. Kasuan, "Model reference adaptive control (MRAC) without integral for glycerin bleaching process," Proc. - 2014 IEEE 10th Int. Colloq. Signal Process. Its Appl. CSPA 2014, pp. 111-116, 2014. a. t. start-up, “model reference control for teamtemperature," pp. 3-8.

[9] A. Landau, I.D., Lozano, R., M’Saad, M., Karimi, Adaptive Control. 2011

[10] C. Pan and J. Lin, "Fuzzy adaptive model reference temperature control of an oil cooler for tool machine," 2004 IEEE Reg. 10 Conf. TENCON 2004., vol. D, pp. 502-505

[11] N. Kasuan, M. N. Taib, and M. H. Fazalul Rahiman, "Model Reference Adaptive Controller to regulate steam temperature in distillation process for essential oil extraction," 2011 IEEE 7th Int. Colloq. Signal Process. its Appl., pp. 298-303, 2011 
[12] S. Pankaj, "Comparative Analysis of MIT Rule and Lyapunov Rule in Model Reference Adaptive Control Scheme," Engineering, vol. 2, no. 4, pp. 154-163, 2011

[13] P. Swarnkar, S. Jain, and R. K. Nema, "Effect of Adaptation Gain in Model Reference Adaptive Controlled Second Order System.," Eng. Technol. Appl. Sci. Res., vol. 1, no. 3, pp. 70-75, 2011

[14] V. Radisavljevic-Gajic, "Linear observers design and implementation," Proc. 2014 Zo. 1 Conf. Am. Soc. Eng. Educ. - "Engineering Educ. Ind. Involv. Interdiscip. Trends", ASEE Zo. 1 2014, 2014

[15] S. H. Hui, S. and Zak, "Observer Design for Systems With Unknown Inputs,” Int. J. Appl. Math. Comput. Sci., vol. 15 , no. 4, pp. 431-446, 2005

[16] T. Kailath, "Linear Systems," Response, vol. 5, no. 6, pp. 928-933, 1980

[17] M. H. A. Jalil, M. N. Taib, and M. H. F. Rahiman, "Back calculation Anti Windup PID controller on Several Well-Known Tuning Method for Glycerin Bleaching Process Temperature Regulation,” vol. 6, no. 3, pp. 39-50, 2014 